This is an electronic reprint of the original article. This reprint may differ from the original in pagination and typographic detail.

\author{
Author(s): Taylor, M.J.; Cullen, David; Procter, M.G.; Bäck, Torbjörn; Cederwall, Bo; Doncel, M.; \\ Braunroth, T.; Dewald, A.; Pakarinen, Janne; Grahn, Tuomas; Greenlees, Paul; \\ Auranen, Kalle; Jakobsson, Ulrika; Julin, Rauno; Juutinen, Sakari; Herzan, Andrej; \\ Konki, Joonas; Leino, Matti; Liotta, R.; Partanen, Jari; Peura, Pauli; Rahkila, Panu; \\ Ruotsalainen, Panu; Sandzelius, Mikael; Sarén, Jan; Sorri, Juha; Stolze, Sanna; Uusitalo, \\ ı..h... 1:un us v. v.. r n \\ Title: $\quad$ Competing single-particle and collective states in the low-energy structure of $113 \mathrm{I}$
}

Year: $\quad 2013$

Version:

Please cite the original version:

Taylor, M.J., Cullen, D., Procter, M.G., Bäck, T., Cederwall, B., Doncel, M., Braunroth, T., Dewald, A., Pakarinen, J., Grahn, T., Greenlees, P., Auranen, K., Jakobsson, U., Julin, R., Juutinen, S., Herzan, A., Konki, J., Leino, M., Liotta, R., . . Xu, F.R. (2013). Competing single-particle and collective states in the low-energy structure of $113 \mathrm{I}$. Physical Review C, 88(5), Article 054307.

https://doi.org/10.1103/PhysRevC.88.054307

All material supplied via JYX is protected by copyright and other intellectual property rights, and duplication or sale of all or part of any of the repository collections is not permitted, except that material may be duplicated by you for your research use or educational purposes in electronic or print form. You must obtain permission for any other use. Electronic or print copies may not be offered, whether for sale or otherwise to anyone who is not an authorised user. 


\title{
Competing single-particle and collective states in the low-energy structure of ${ }^{113}$ I
}

\author{
M. J. Taylor, ${ }^{1, *}$ D. M. Cullen, ${ }^{1}$ M. G. Procter, ${ }^{1}$ T. Bäck, ${ }^{2}$ B. Cederwall,,${ }^{2}$ M. Doncel, ${ }^{2}$ T. Braunroth, ${ }^{3}$ A. Dewald, ${ }^{3}$ \\ J. Pakarinen, ${ }^{4}$ T. Grahn, ${ }^{4}$ P. T. Greenlees ${ }^{4}$ K. Auranen ${ }^{4}$ U. Jakobsson, ${ }^{4}$ R. Julin, ${ }^{4}$ S. Juutinen, ${ }^{4}$ A. Herzáñ,${ }^{4}$ J. Konki, ${ }^{4}$ \\ M. Leino, ${ }^{4}$ R. Liotta, ${ }^{4}$ J. Partanen, ${ }^{4}$ P. Peura, ${ }^{4}$ P. Rahkila, ${ }^{4}$ P. Ruotsalainen, ${ }^{4}$ M. Sandzelius, ${ }^{4}$ J. Sarén, ${ }^{4}$ J. Sorri, ${ }^{4}$ S. Stolze, ${ }^{4}$ \\ J. Uusitalo, ${ }^{4}$ W. Y. Liang, ${ }^{5}$ and F. R. $\mathrm{Xu}^{5}$ \\ ${ }^{1}$ School of Physics and Astronomy, The University of Manchester, Oxford Road, Manchester, M13 9PL, United Kingdom \\ ${ }^{2}$ Department of Physics, Royal Institute of Technology, Stockholm, SE-10691, Sweden \\ ${ }^{3}$ Institut für Kernphysik, Universität zu Köln, Köln, D-50937, Germany \\ ${ }^{4}$ Department of Physics, University of Jyväskylä, Jyväskylä, FIN-40014, Finland \\ ${ }^{5}$ School of Physics, Peking University, Beijing 100871, China \\ (Received 17 September 2013; published 7 November 2013)
}

\begin{abstract}
To understand the low-energy structure of the neutron deficient iodine isotopes, lifetimes for the low-lying $9 / 2^{+}$and $11 / 2^{+}$positive-parity states in ${ }^{113}$ I have been measured as $\tau=28(4)$ ps and $\tau=3.7(7)$ ps, respectively. The lifetime for the $11 / 2^{-}$state, which feeds the $9 / 2^{+}$and $11 / 2^{+}$states, was remeasured with improved accuracy as $\tau=216(7) \mathrm{ps}$. The reduced transition probability, $B(E 2)=32(5)$ W.u., for the $9 / 2^{+} \rightarrow 5 / 2^{+}$transition agrees with that calculated within the shell model using a Hamiltonian based on the charge-dependent Bonn nucleon-nucleon interaction. In contrast, the much larger transition probability, $B(E 2)=209(39)$ W.u., measured for the $11 / 2^{+} \rightarrow 7 / 2^{+}$transition has been interpreted, with the aid of configuration-constrained total Routhian surface calculations, as resulting from a slightly $\gamma$-soft rotor with an associated quadrupole deformation of $\beta_{2} \approx 0.18$. Remarkably similar reduced $E 1$ transition probabilities of $5.5(5) \times 10^{-4}$ and $4.9(5) \times 10^{-4}$ W.u. were deduced for the $11 / 2^{-} \rightarrow 9 / 2^{+}$and $11 / 2^{-} \rightarrow 11 / 2^{+}$transitions, respectively, which feed apparently dissimilar but competing structures.
\end{abstract}

DOI: 10.1103/PhysRevC.88.054307

PACS number(s): 21.60.Cs, 21.10.Tg, 27.60.+j

\section{INTRODUCTION}

The systematic comparison of reduced transition probabilities, determined from lifetime measurements, can reveal trends in the evolution of nuclear shapes in isotopes spanning regions of single-particle and collective behavior. Reduced transition rates between the ground and first excited $2^{+}$states in the even- $A \mathrm{Sn}$ isotopes showed excellent agreement with those calculated within the framework of the shell model using a Hamiltonian based on the the state-of-the-art chargedependent Bonn (CD-Bonn) nucleon-nucleon interaction [1]. Although the best agreement was observed when a ${ }^{100} \mathrm{Sn}$ core was adopted, a deviation was observed for isotopes with $N<66$ which has been interpreted as an increase in collectivity as the $N=Z=50$ doubly-magic shell closure is approached [2-5]. However, any such increase in collectivity or weakening of the $N=50$ shell closure currently lacks a stringent theoretical interpretation [6].

The recently determined $B\left(E 2 ; 0^{+} \rightarrow 2^{+}\right)=0.10(4) e^{2} \mathrm{~b}^{2}$ value for ${ }^{104} \mathrm{Sn}$, using the PRESPEC setup at GSI [7], is significantly lower than that measured for ${ }^{106} \mathrm{Sn}$ [5] and heavier isotopes [2-5] and shows that the previously proposed anomalous $B(E 2)$ values for the lightest tin isotopes should be treated with caution. The measured $B(E 2)$ for ${ }^{104} \mathrm{Sn}$ is well reproduced by shell-model calculations indicating a fairly robust $N=Z=50$ shell closure. In addition, the measured $B\left(E 2 ; 2^{+} \rightarrow 0^{+}\right)$value for ${ }^{108} \mathrm{Te}$ [8] indicates that no enhancement of collectivity relative to the standard large-

*m.j.taylor@manchester.ac.uk scale shell model (LSSM) predictions was found for this $N=56$ nucleus. Further spectroscopic data in neighboring nuclei, such as the neutron deficient iodine isotopes, may help to shed some light on this important issue and provides the motivation for the present work.

In a previous study of ${ }^{113}$ I, Starosta et al. [9] observed excited states to very high spin $(\sim 60 \hbar)$ thus establishing a comprehensive decay scheme. In subsequent work, Petkov et al. [10] measured lifetimes for some of the negative-parity states in the yrast band; however, lifetimes were not reported for the low-lying positive-parity states. Lifetime data for the yrast $9 / 2^{+}$state in the odd- $A$ isotopes is only available for $A>$ 113 and the only other measurement for a low-lying $11 / 2^{+}$ state in these isotopes is ${ }^{109} \mathrm{I}$ which was performed by this collaboration [11]. For ${ }^{109} \mathrm{I}$, the $11 / 2^{+} \rightarrow 7 / 2^{+}$experimental reduced transition probability was found to be considerably smaller than that predicted by shell-model calculations using the CD-Bonn nucleon-nucleon potential. As ${ }^{109} \mathrm{I}$ is unbound to proton decay, the discrepancy was explained by the inability of the shell-model calculations to accurately account for the behavior of the unbound nuclear states [11]. It was clear from the previous studies of the tin and iodine isotopes that further spectroscopic information on the low-energy structure of ${ }^{113} \mathrm{I}$ was needed.

\section{EXPERIMENTAL DETAILS AND DATA ANALYSIS}

The recoil distance Doppler shift (RDDS) technique [12] has proven to be an extremely successful method for determining the lifetimes of excited nuclear states down to $\sim 1$ ps. 
When sufficient statistics are available, the use of coincident $\gamma$-ray transitions have allowed the effects of unobserved side feeding to be eliminated from the lifetime derivation, yielding a highly accurate measurement of the states of interest.

Excited states in ${ }^{113} \mathrm{I}$ were populated using the ${ }^{58} \mathrm{Ni}\left({ }^{58} \mathrm{Ni}, 3 p\right)$ reaction at a beam energy of $210 \mathrm{MeV}$. The K130 cyclotron, at the Accelerator Laboratory of the University of Jyväskylä, Finland, accelerated the ${ }^{58} \mathrm{Ni}$ beam onto a $1.05 \mathrm{mg} / \mathrm{cm}^{2}{ }^{58} \mathrm{Ni}$ target. The target, along with a $1.2 \mathrm{mg} / \mathrm{cm}^{2} \mathrm{Mg}$ degrader foil, were mounted within the Differential Plunger for Unbound Nuclear States (DPUNS) [13] at the center of the Jurogam-II $\gamma$-ray spectrometer [14]. The DPUNS degrader foil acted to reduce the full velocity of the recoiling reaction products from $v / c=0.040$ (1) to $v / c=0.025(1)$. DPUNS was operated in "gas mode" with the recoil ion transport unit (RITU) $[15,16]$ being used as a beam dump. The helium gas of RITU was extracted from the beamline by a Roots differential pumping system (see Ref. [13] for further information). Prompt $\gamma$ rays de-exciting the ${ }^{113} \mathrm{I}$ nuclei were recorded by the Jurogam-II array which comprised 15 Eurogam Phase-1-type single-crystal germanium detectors, located at backward angles of $157.6^{\circ}$ (5 ring 1 detectors) and $133.6^{\circ}$ (10 ring 2 detectors) to the beam axis and 24 clover detectors located in two detector rings (rings 3 and 4) either side of $90^{\circ}\left( \pm 14.5^{\circ}\right)$. All $\gamma$ rays detected by the Jurogam-II detectors were time stamped by a $100 \mathrm{MHz}$ clock from the total data readout acquisition system [17]. Data were sorted into one-dimensional histograms and two-dimensional matrices for offline analysis. The lifetimes of the low-lying states in ${ }^{113} \mathrm{I}$ were determined using the differential decay curve method (DDCM) [18]. In the present work, the lifetime of the level of interest, $\tau(x)$, was derived at each target-to-degrader distance $x$ using the relationship

$$
\tau(x)=\frac{\left\{I_{B}^{s}, I_{A}^{d}\right\}}{\frac{d}{d x}\left\{I_{B}^{s}, I_{A}^{s}\right\}} \frac{1}{v},
$$

where $I_{A}^{s}$ and $I_{A}^{d}$ correspond to the normalized intensities of the shifted $(s)$ and degraded $(d)$ components of the depopulating transition that correlate with the shifted component $\left(I_{B}^{S}\right)$ of the transition feeding the state of interest. The intensities were normalized across each distance to the number of $\gamma$ rays in the corresponding, ungated, total projection. Finally, $v$ is the mean velocity of the recoiling nuclei. The lifetime of the state under investigation is then determined from a piecewise fit to the normalized shifted intensity data. The derivative of this function, multiplied by the value of the lifetime $\tau(x)$ is simultaneously fitted to the denominator in Eq. (1) and a $\chi^{2}$ minimum found. The weighted average of each individual lifetime value, within the so-called "region of sensitivity" [19], was used to deduce the final lifetime value.

\section{RESULTS}

Figure 1(a) shows an ungated $\gamma$-ray spectrum obtained from a Jurogam-II clover versus clover $\gamma-\gamma$ matrix (ring 3 versus ring 4 detectors). The most prominent yrast decays in ${ }^{113} \mathrm{I}$ are denoted by their energies. The most intense decays originating from other reaction channels are denoted by symbols. A

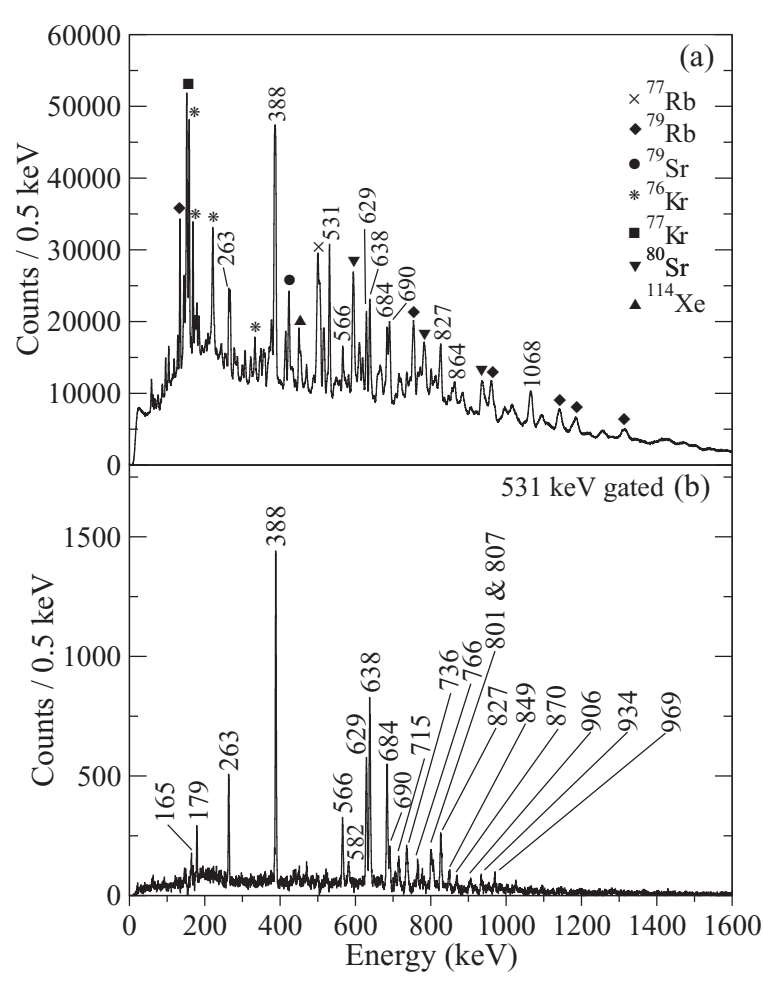

FIG. 1. $\gamma$-ray spectra obtained from a Jurogam-II clover vs clover $\gamma-\gamma$ matrix. (a) Total (ungated) projection with the energies of the ${ }^{113} \mathrm{I}$ transitions that are of particular relevance to this work highlighted. Transitions marked with symbols originate from nuclei produced in reactions on the target $\left({ }^{114} \mathrm{Xe}\right)$ and $\mathrm{Mg}$ degrader foil $(A \sim 80)$. (b) $531 \mathrm{keV}$ gated spectrum with ${ }^{113} \mathrm{I}$ transitions labeled with their energy.

$450 \mathrm{keV}$ transition from ${ }^{114} \mathrm{Xe}(2 p$ channel) can be seen along with numerous decays from $A \approx 80$ nuclei produced in ${ }^{58} \mathrm{Ni}$ reactions on the $\mathrm{Mg}$ degrader foil. Figure 1(b) was created from the same $\gamma-\gamma$ matrix as that used for Fig. 1(a) and shows decays that correlate with the $531 \mathrm{keV} 15 / 2^{-} \rightarrow 11 / 2^{-}$transition in ${ }^{113} \mathrm{I}$. All of the transitions in Fig. 1(b) have been identified as belonging to ${ }^{113} \mathrm{I}[9,10]$.

Previous studies of ${ }^{113} \mathrm{I}$ managed to establish a comprehensive level scheme to high spins [9], thus the $\gamma-\gamma$ coincidence matrix produced in this work was only used to confirm the transition intensities and low-spin feeding patterns. Figure 2 shows the resulting partial level scheme highlighting the states and transitions that are of particular importance to the subsequent excited-state lifetime analysis. The $\gamma$-ray intensities shown in Fig. 2 have been efficiency corrected and are summarized along with the transition energies in Table I.

\section{A. Negative-parity band}

Previous work by Petkov et al. [10], measured lifetimes for some of the states in the negative-parity yrast band which is understood to be built upon a low- $K$ prolate $\pi h_{11 / 2}$ orbital. In particular, an accurate measurement of the $15 / 2^{-}$state lifetime, $\tau=7.22(43) \mathrm{ps}$, was made [10]. A remeasurement of this lifetime was performed with the current data set to reveal any potential systematic errors associated with the 


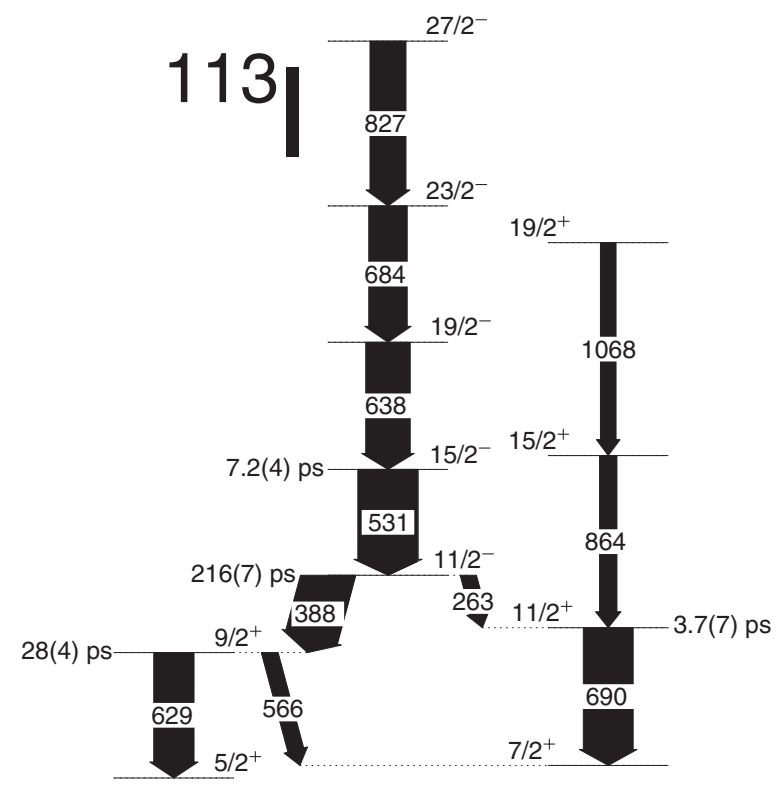

FIG. 2. Partial level scheme for ${ }^{113}$ I, produced from a $\gamma-\gamma$ correlation matrix, showing the states and transitions that are of particular importance in this work. The observed correlations confirm the transition ordering reported in Refs. [9,10]. The state lifetimes shown are those measured in this work.

experimental setup. For excited-state lifetime determination a $\gamma-\gamma$ correlation matrix was produced using data recorded in the Jurogam-II ring $1\left(157.5^{\circ}\right)$ against ring $2\left(137.5^{\circ}\right)$ detectors. The large angles, relative to the recoil direction, that these detectors subtend, maximizes the separation between the shifted and degraded components of the photopeak. By gating on the shifted component of the $638 \mathrm{keV}$ transition (ring 2) and projecting onto the ring 1 axis, the intensities of the $531 \mathrm{keV}$ shifted and degraded components were measured as a function of target-to-degrader distance. Figure 3 shows the resulting ring 1 spectra for four target-to-degrader distances ranging from 62 to $760 \mu \mathrm{m}$. The shifted (left peak) and degraded (right peak) intensity change is clear with increasing distance, with the $531 \mathrm{keV}$ decay being fully shifted by $760 \mu \mathrm{m}$. The Gaussian fits used to extract the relative intensities are

TABLE I. ${ }^{113}$ I efficiency corrected $\gamma$-ray intensities measured in this work for the transitions shown in Fig. 2. Intensities have been normalized to the $531 \mathrm{keV} 15 / 2^{-} \rightarrow 11 / 2^{-}$transition.

\begin{tabular}{lcc}
\hline \hline$J^{\pi}$ & $E_{\gamma}(\mathrm{keV})$ & $I_{\gamma}(\%)$ \\
\hline $11 / 2^{-}$ & $263.11(5)$ & $26(2)$ \\
$11 / 2^{-}$ & $388.01(7)$ & $89(6)$ \\
$15 / 2^{-}$ & $530.85(2)$ & $100(1)$ \\
$9 / 2^{+}$ & $566.10(11)$ & $27(4)$ \\
$9 / 2^{+}$ & $629.03(3)$ & $67(2)$ \\
$19 / 2^{-}$ & $637.84(11)$ & $74(1)$ \\
$23 / 2^{-}$ & $683.90(5)$ & $64(2)$ \\
$11 / 2^{+}$ & $690.48(5)$ & $83(2)$ \\
$27 / 2^{-}$ & $826.64(30)$ & $60(7)$ \\
$15 / 2^{+}$ & $863.50(15)$ & $29(2)$ \\
$19 / 2^{+}$ & $1068.05(7)$ & $61(6)$ \\
\hline \hline
\end{tabular}

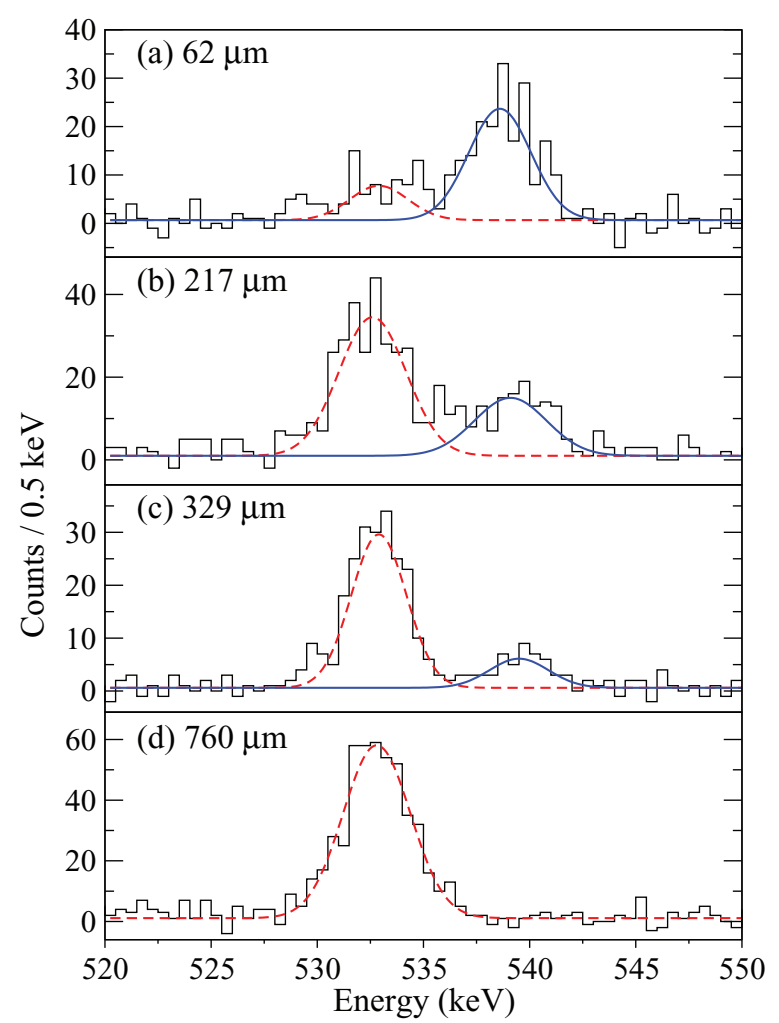

FIG. 3. (Color online) $638 \mathrm{keV}$ (ring 2) gated spectra, recorded by the Jurogam-II ring $1\left(\theta=157^{\circ}\right)$ detectors, showing the shifted (left peak) and degraded (right peak) components of the $531 \mathrm{keV}$ transition, from the $15 / 2^{-}$state for target-to-degrader distances of (a) 61.7, (b) 216.6, (c) 329.2, and (d) $759.8 \mu \mathrm{m}$. Gaussian fits used to extract the component intensities are also shown (dashed and solid lines).

also shown. The measured intensities were normalized to the number of counts in the ring 1 total projections. A simultaneous fit to the shifted and degraded data was performed and the result used to evaluate the state lifetime at each target-to-degrader distance in the region of sensitivity. A weighted mean resulted in a lifetime of 7.21(43) ps, in excellent agreement with that measured previously, 7.22(43) ps [10]. The agreement between the literature value for the $15 / 2^{-}$state lifetime and that measured in this work shows that no significant systematic errors are present on any lifetime values extracted from these data.

The current literature value for the lifetime of the $11 / 2^{-}$ state, $\tau=229(52)$ ps [10], has a 23\% uncertainty, prompting a remeasurement using this data set. The analysis for the $11 / 2^{-}$ state lifetime is analogous with that performed for the $15 / 2^{-}$ state. A ring 2 gate on the shifted component of the $531 \mathrm{keV}$ transition was used to produce a ring 1 spectrum containing the shifted and degraded components of the $388 \mathrm{keV}$ decay. Figure 4(a) shows the $11 / 2^{-}$state lifetime evaluated for targetto-degrader distances in the region-of-sensitivity along with the weighted mean of $\tau=216(7)$ ps. Figure 4(b) shows the normalized shifted intensities along with the result of a leastsquares fit (solid line) which had a $\chi^{2}$ value of 1.25 . The newly measured value is consistent with the current literature value but has a much improved uncertainty $(\sim 3 \%)$. 


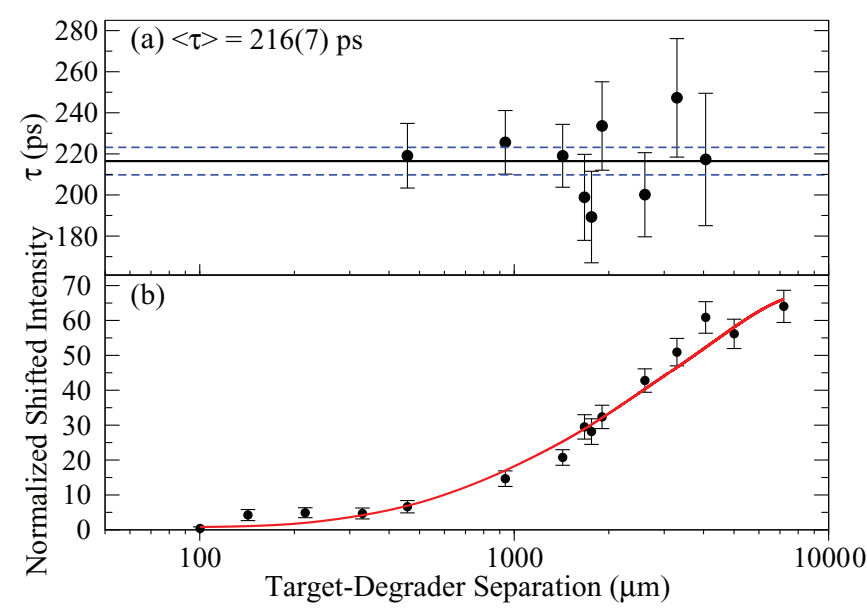

FIG. 4. (Color online) (a) The lifetime of the $11 / 2^{-}$state evaluated for each target-degrader separation in the region-of-sensitivity along with the weighted mean of 216 ps (solid line) and its uncertainty $\pm 7 \mathrm{ps}$ (dashed lines). (b) Normalized shifted intensities for the $11 / 2^{-} \rightarrow 9 / 2^{+} 388 \mathrm{keV}$ decay along with the results of a least-squares fit (solid line).

\section{B. $11 / 2^{+}$state lifetime}

The previous RDDS study of ${ }^{113}$ I did not report on any measurement of the lifetimes of the low-lying positive-parity states that are populated by decays out of the $11 / 2^{-}$band head. In the present work, a measurement of the positiveparity $11 / 2^{+}$state was performed using a direct gate on the shifted component of $864 \mathrm{keV}$ decay (ring 2) and measuring the component intensities of the $690 \mathrm{keV}$ transition in the ring 1 projection. A gate on the $263 \mathrm{keV}$ feeding transition could not be used in this analysis, because the degraded component of the $684 \mathrm{keV} 23 / 2^{-} \rightarrow 19 / 2^{-}$transition, present in the decay path, interferes with the shifted component of the $690 \mathrm{keV}$ decay that depopulates the $11 / 2^{+}$state. Figure 5 shows the resulting ring 1 spectra for three target-to-degrader distances ranging from $7 \mu \mathrm{m}$ to $10 \mathrm{~mm}$. The relative change in the shifted (left peak) and degraded (right peak) photopeak intensities with increasing distance is clear with the $690 \mathrm{keV}$ decay being fully shifted at $10 \mathrm{~mm}$. The Gaussian fits used to extract the relative intensities are also shown.

Figure 6(a) shows the $11 / 2^{+}$state lifetime evaluated for target-to-degrader distances in the region-of-sensitivity along with the weighted mean of $\tau=3.7(7)$ ps. Figure 6(b) shows the normalized shifted intensities along with the result of a least-squares fit (solid line) which had a $\chi^{2}$ value of 0.35 .

\section{C. $9 / 2^{+}$state lifetime}

The $9 / 2^{+}$state lifetime spectra could not be obtained by gating on a direct feeding transition (as was used for the $15 / 2^{-}, 11 / 2^{-}$, and $11 / 2^{+}$states) due to contamination from higher-lying transitions. Instead, a gate was placed on the degraded component [20] of the $629 \mathrm{keV} \mathrm{9/2} \rightarrow 5 / 2^{+}$decay and the shifted and degraded intensities of the populating 388 $\mathrm{keV}$ decay were measured. Figure 7 shows the resulting ring 1 spectra for three target-to-degrader distances ranging from

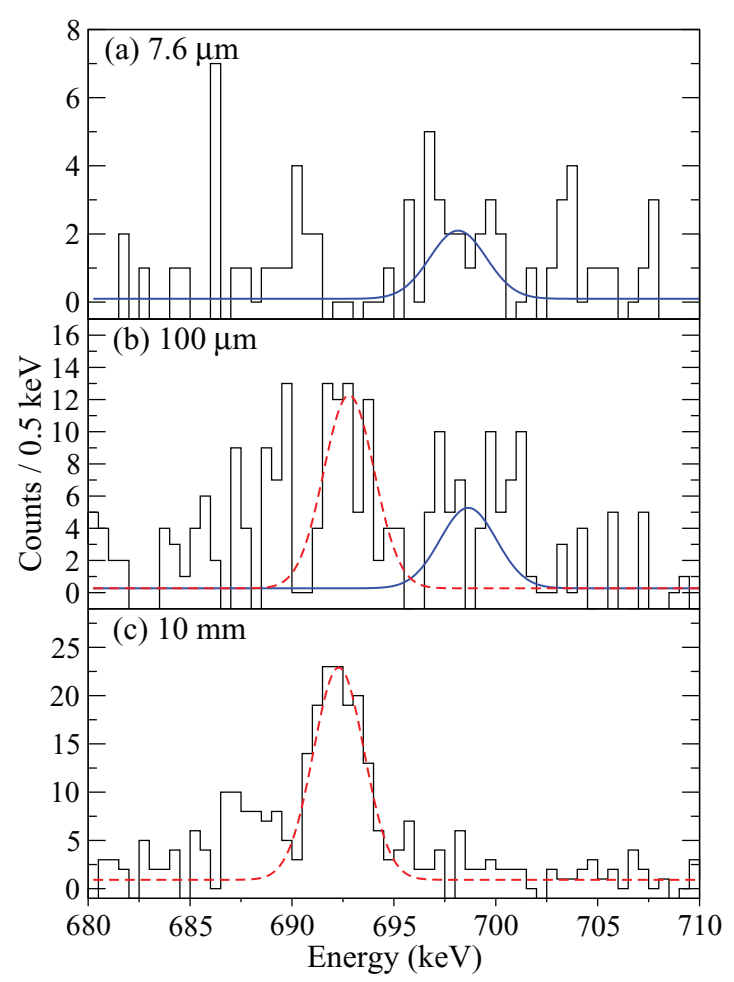

FIG. 5. (Color online) $864 \mathrm{keV}$ (ring 2) gated spectra, recorded by the Jurogam-II ring $1\left(\theta=157^{\circ}\right)$ detectors, showing the shifted (left peak) and degraded (right peak) components of the $690 \mathrm{keV}$ transition, from the $11 / 2^{+}$state in ${ }^{113} \mathrm{I}$, for target-to-degrader distances of (a) 7.6, (b) 100.3, and (c) $9976 \mu \mathrm{m}$. Gaussian fits used to extract the component intensities are also shown (dashed and solid lines).

$217 \mu \mathrm{m}$ to $10 \mathrm{~mm}$. The Gaussian fits used to extract the relative intensities are also shown.

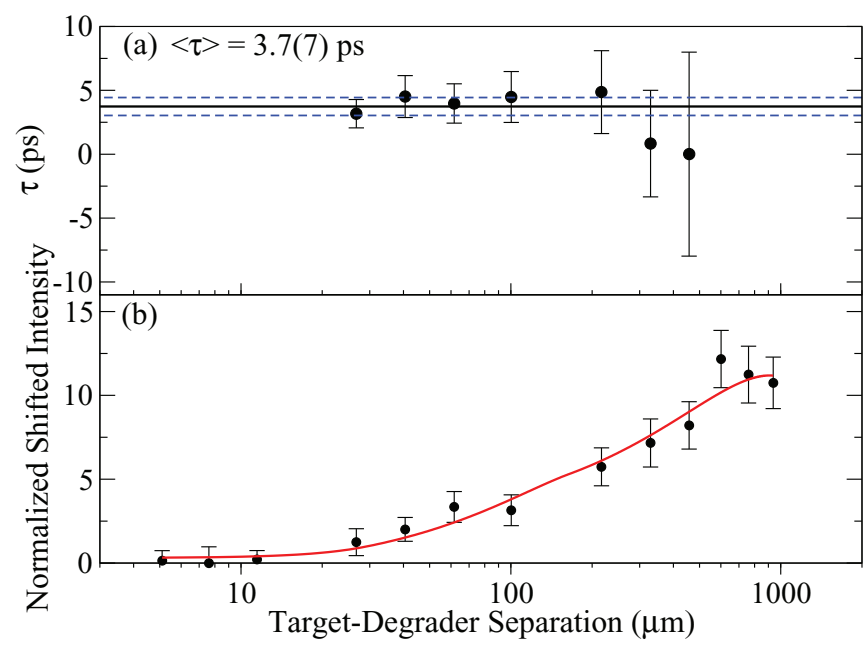

FIG. 6. (Color online) (a) The lifetime of the $11 / 2^{+}$state evaluated for each target-degrader separation in the region-of-sensitivity along with the weighted mean of $3.7 \mathrm{ps}$ (solid line) and its uncertainty \pm 0.7 ps (dashed lines). (b) Normalized shifted intensities for the $11 / 2^{+} \rightarrow 7 / 2^{+} 690 \mathrm{keV}$ decay along with the results of a leastsquares fit (solid line). 


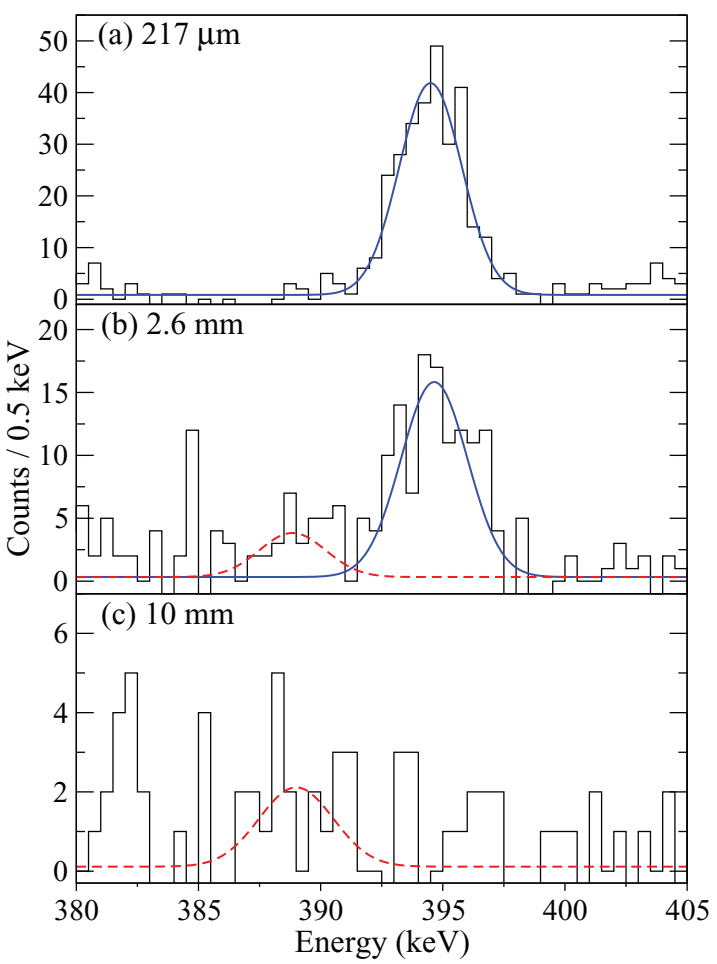

FIG. 7. (Color online) $629 \mathrm{keV}$ (ring 2) degraded component gated spectra, recorded by the Jurogam-II ring $1\left(\theta=157^{\circ}\right)$ detectors, showing the shifted (left peak) and degraded (right peak) components of the $388 \mathrm{keV}$ transition, feeding the $9 / 2^{+}$state, for target-todegrader distances of (a) 216.6, (b) 2603, and (c) $9976 \mu \mathrm{m}$. Gaussian fits used to extract the component intensities are also shown (dashed and solid lines).

Figure 8 (a) shows the $9 / 2^{+}$state lifetime evaluated for target-to-degrader distances in the region-of-sensitivity along with the weighted mean of $\tau=28(4)$ ps. Figure 8(b) shows the normalized shifted intensities along with the result of a least-squares fit (solid line) which had a $\chi^{2}=1.6$.

\section{DISCUSSION}

Reduced quadrupole transition probabilities can be deduced from measured state lifetimes using

$$
B(E 2)=\frac{817.66}{E_{\gamma}^{5} \tau(1+\alpha)} e^{2} \mathrm{fm}^{4},
$$

where $E_{\gamma}$ is in $\mathrm{MeV}, \tau$ is in picoseconds, and the $(1+\alpha)$ term corrects for internal conversion. Using Eq. (2) with the newly measured lifetimes for the $9 / 2^{+}$and $11 / 2^{+}$states, transition probabilities of $212(33) e^{2} \mathrm{fm}^{4}$ [32(5) W.u.] and 1403(265) $e^{2} \mathrm{fm}^{4}$ [209(39) W.u.] were deduced, respectively. The $B(E 2)$ value for the $11 / 2^{+}$state is indicative of collectivity, possibly arising from the rotation of a prolate deformed shape, whereas the relatively lower value for the $9 / 2^{+}$state hints at a more single-particle structure. To interpret these findings, shell-model calculations were performed in this work.

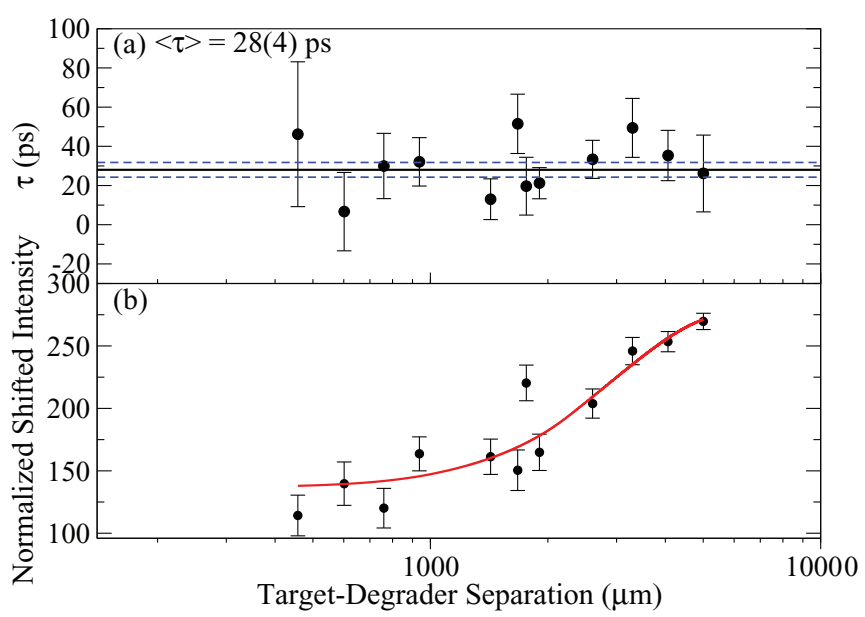

FIG. 8. (Color online) (a) The lifetime of the $9 / 2^{+}$state evaluated for each target-degrader separation in the region-of-sensitivity along with the weighted mean of $28 \mathrm{ps}$ (solid line) and its uncertainty \pm 4 ps (dashed lines). (b) Normalized shifted intensities for the $11 / 2^{-} \rightarrow 9 / 2^{+} 388 \mathrm{keV}$ decay along with the results of a leastsquares fit (solid line).

\section{A. Shell-model calculations}

In a simple shell-model picture one may expect the lowenergy structure of ${ }^{113} \mathrm{I}$ to arise from configurations dominated by the three valence protons outside of the $Z=50$ close shell. The unpaired proton predominantly residing in the $d_{5 / 2}$ orbital and the remaining two protons (and the ten valence neutrons outside of the $N=50$ closed shell) coupled to spin zero would constitute the $5 / 2^{+}$ground state. Starting from this simple picture, the first excited $9 / 2^{+}$state could then be created from a recoupling of a pair of spin-zero coupled protons to spin 2. The observed $7 / 2^{+}$state, however, cannot be explained in this way. A significant occupation of another orbital, such as the $g_{7 / 2}$, needs to be invoked in order to create an excited $7 / 2^{+}$state. The observation of the $566 \mathrm{keV}$ decay from the $9 / 2^{+}$state may suggest that the $g_{7 / 2}$ orbital plays a significant role in the generation of the $9 / 2^{+}$state, because a proton or neutron pair recoupling back to spin zero could not produce the final $7 / 2^{+}$state. A more likely scenario is that the $9 / 2^{+}$state arises from a pair of spin-2 coupled protons predominately occupying the $g_{7 / 2}$ orbital as opposed to the $d_{5 / 2}$ orbital. The $11 / 2^{+}$state could then be understood in terms of the odd proton occupying the $g_{7 / 2}$ orbital along with a spin-2 coupled proton pair. It is therefore evident that the low-lying positive-parity states of interest in this work could be explained by a simple shell-model picture involving only the $d_{5 / 2}$ and $g_{7 / 2}$ orbitals and the recoupling of proton spins to zero or two.

To test this hypothesis, shell-model calculations using the code NUSHELL@MSU [21] were performed to help interpret the newly measured $B(E 2)$ for the $629 \mathrm{keV} 9 / 2^{+} \rightarrow 5 / 2^{+}$ transition. The 'sn100pn' Hamiltonian [22] was used with a model space containing the $1 g_{7 / 2}, 2 d_{5 / 2}, 2 d_{3 / 2}, 3 s_{1 / 2}$, $1 h_{11 / 2}$ proton and neutron orbitals outside a ${ }^{100} \mathrm{Sn}$ core. Proton single-particle energies of $-9.68,-8.72,-7.24$, and $-6.88 \mathrm{MeV}$ where used along with neutron single-particle energies of $-9.74,-8.97,-7.31,-7.62$, and $-7.38 \mathrm{MeV}$ 


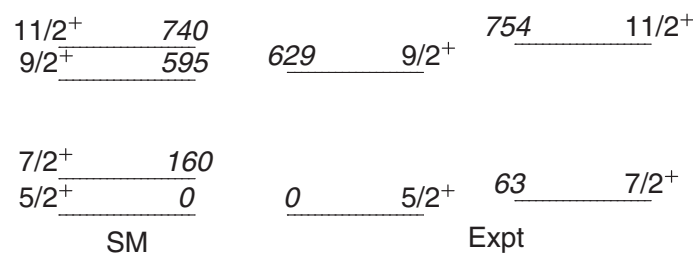

FIG. 9. Comparison between the experimentally determined level energies for the low-lying positive-parity states and those calculated within the framework of the shell model. Calculations were performed with the code NUSHELL@MSU [21] utilizing the 'sn100pn' Hamiltonian [22] with an effective interaction derived from the CD-Bonn nucleon-nucleon potential $[1,23]$.

for the $1 g_{7 / 2}, 2 d_{5 / 2}, 2 d_{3 / 2}$, and $1 h_{11 / 2}$ orbitals, respectively. The residual two-body interaction was obtained from a $G$ matrix derived from the CD-Bonn nucleon-nucleon potential $[1,23]$. The aforementioned arguments were used to restrict the neutron and proton occupations; all nucleons were restricted to the $d_{5 / 2}$ and $g_{7 / 2}$ orbitals with neutrons filling the $d_{5 / 2}$ orbital. The calculated energy eigenvalues are compared to those determined experimentally in Fig. 9. Although the shell-model overestimates the excitation energy of the $7 / 2^{+}$ state, good agreement is observed between the calculated and experimental partial level schemes.

Using the calculated wave functions, the quadrupole reduced transition probability for the $9 / 2^{+} \rightarrow 5 / 2^{+}$transition was determined. A Woods-Saxon potential along with standard effective proton and neutron charges of $1.5 e$ and $0.5 e$, respectively, were adopted. A $B(E 2)$ value of 45 W.u. was calculated for the $629 \mathrm{keV}$ transition which agrees, within $3 \sigma$, with the $B(E 2)=32(5)$ W.u. measured in this work. An intrinsic quadrupole moment of $-0.12 \mathrm{eb}$ was also calculated for the $9 / 2^{+}$state. The dominant wave-function configurations for the excited $7 / 2^{+}, 9 / 2^{+}$, and $11 / 2^{+}$states are represented graphically in Fig. 10. The calculated wave function for the $9 / 2^{+}$state exhibits a significant $g_{7 / 2}$ contribution, as postulated. The similarity between the configurations for the

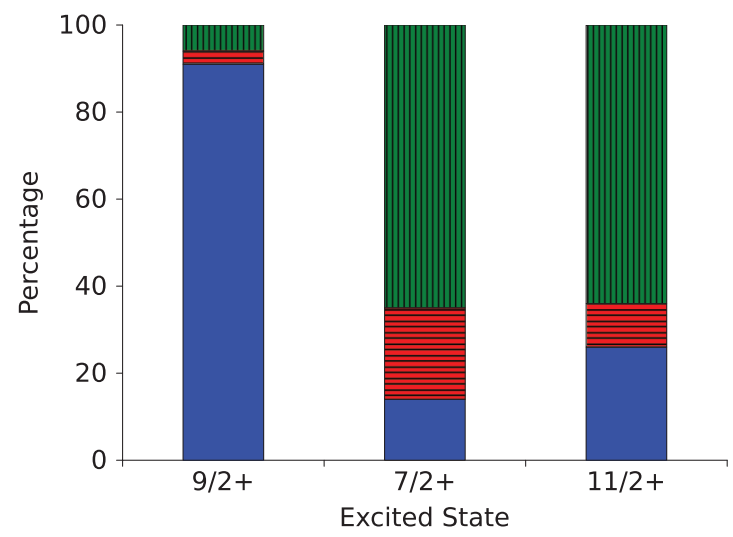

FIG. 10. (Color online) Graphical representation of the shellmodel calculated wave functions for the low-lying excited $9 / 2^{+}$, $7 / 2^{+}$, and $11 / 2^{+}$states in ${ }^{113} \mathrm{I}$. The bottom (blue with no pattern), middle (red with horizontal lines), and top (green with vertical lines) portions of each bar denote the $\pi g_{7 / 2}^{2} d_{5 / 2}^{1}, \pi g_{7 / 2}^{1} d_{5 / 2}^{2}$, and $\pi g_{7 / 2}^{3}$ contributions, respectively.
TABLE II. Summary of the lifetimes and reduced quadrupole transition probabilities measured in this work. Shell-model calculated transition probabilities are also shown.

\begin{tabular}{lcccc}
\hline \hline$J_{i}^{\pi} \rightarrow J_{f}^{\pi}$ & $\begin{array}{c}\mathrm{E}_{\gamma} \\
(\mathrm{keV})\end{array}$ & $\begin{array}{c}\tau \\
(\mathrm{ps})\end{array}$ & $\begin{array}{c}B(E 2)_{\exp } \\
\text { (W.u.) }\end{array}$ & $\begin{array}{c}B(E 2)_{\mathrm{SM}} \\
\text { (W.u.) }\end{array}$ \\
\hline $11 / 2^{+} \rightarrow 7 / 2^{+}$ & $690.5(5)$ & $3.7(7)$ & $209(39)$ & 59 \\
$9 / 2^{+} \rightarrow 5 / 2^{+}$ & $629.0(5)$ & $39(6)^{\mathrm{a}}$ & $32(5)$ & 45 \\
\hline \hline
\end{tabular}

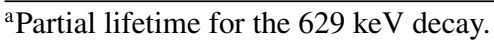

$7 / 2^{+}$and $11 / 2^{+}$states is clear from Fig. 10 as is the difference between those configurations and that of the $9 / 2^{+}$state.

$B(E 2)=59$ W.u. was calculated for the $11 / 2^{+} \rightarrow 7 / 2^{+}$ $690 \mathrm{keV}$ transition, which is considerably less than the experimental value of 209(39). The measured and calculated reduced transition probabilities are summarized in Table II. It is interesting to note that the shell-model overestimated the experimental $B\left(E 2: 11 / 2^{+} \rightarrow 7 / 2^{+}\right)=710(90) e^{2} \mathrm{fm}^{4}$ $\left[B(E 2)_{\mathrm{SM}}=1146 e^{2} \mathrm{fm}^{4}\right]$ for ${ }^{109} \mathrm{I}$, whereas the shell model underestimates the corresponding $B(E 2)$ for ${ }^{113} \mathrm{I}$.

\section{B. $11 / 2^{+}$state}

In the limit of rigid rotation the transition quadrupole moment $Q_{t}$ equals the intrinsic quadrupole moment $Q_{0}$. The intrinsic quadrupole moment of the $11 / 2^{+}$state can, therefore, be determined within the framework of the rigid rotor model [24], using

$$
B(E 2: I \rightarrow I-2)=\frac{5}{16 \pi} Q_{t}^{2}\langle\mathrm{IK} 20 \mid \mathrm{I}-2 \mathrm{~K}\rangle^{2} .
$$

Examination of the positive-parity Nilsson orbitals near to the Fermi surface revealed that the unpaired proton could occupy the $3 / 2$ [422] or the $1 / 2$ [431] Nilsson states from the $g_{7 / 2}$ orbital or the $1 / 2$ [420] state from the $d_{5 / 2}$ orbital leading to possible values for the $K$ quantum number of $3 / 2$ or $1 / 2$. For $K=3 / 2$ the intrinsic quadrupole moment, deduced from the measured $B(E 2)$, is $2.35(22) e$ b and for $K=1 / 2$, $Q_{0}=2.16(20) e$ b. These quadrupole moments correspond to quadrupole deformation parameters of $\beta_{2}=0.17(2)$ and $\beta_{2}=$ 0.16 (1) for $K=3 / 2$ and $K=1 / 2$, respectively, evaluated using

$$
Q_{0}=\frac{3}{\sqrt{5 \pi}} Z R_{0}^{2} \beta_{2}\left(1+\frac{1}{8} \sqrt{\frac{5}{\pi}} \beta_{2}\right),
$$

assuming $\gamma=0^{\circ}$.

\section{C. $11 / 2^{-}$state}

The measured transition probabilities and shell-modelcalculated wave functions show a clear difference between the structures of the $11 / 2^{+}$and $9 / 2^{+}$states. This difference should also manifest itself in the $E 1$ transitions that feed into these structures from the $11 / 2^{-}$state. Taking into account a weak $179 \mathrm{keV}$ decay to a second $9 / 2_{2}^{+}$state [10], partial lifetimes of 304(25) and 1075(94) ps were deduced for the 388 and 
$263 \mathrm{keV}$ decays, respectively, using the 216(7) ps lifetime for the $11 / 2^{-}$state remeasured in this work. Using the deduced partial lifetimes and conversion coefficients taken from BrIcc [25], $B(E 1)$ values of $5.5(5) \times 10^{-4}$ and $4.9(5) \times 10^{-4}$ W.u. were calculated for the 388 and $263 \mathrm{keV}$ decays, respectively. The similarity between the decay probabilities for the 388 and $263 \mathrm{keV}$ transitions, assumed to be populating states with different structures, is startling. This similarity was alluded to, but not discussed in detail, in the previous study of ${ }^{113} \mathrm{I}$ by Petkov and co-workers [10]. In that study the lifetimes of the $15 / 2^{-}$and $19 / 2^{-}$states were measured along with that of the $11 / 2^{-}$state. The deduced transition probabilities were explained using the rigid rotor model assuming incomplete alignment of the odd proton. The best description of the data was obtained using quadrupole deformation parameters in the range $\beta_{2}=0.24-0.26$. The quadrupole deformation for the $11 / 2^{-}$state is much larger than that for the $11 / 2^{+}$state. This difference in deformation could be a manifestation of a change in shape between the $11 / 2^{-}$and $11 / 2^{+}$states which would hinder the decay somewhat. To try and understand the origin of this similarity between strengths for transitions populating two apparently different structures, configuration-constrained total Routhian surface calculations have been performed in this work.

\section{Configuration-constrained total Routhian surface calculations}

Total Routhian surface (TRS) calculations provide a theoretical means of describing collective states in rotating nuclei. As the extent of nuclear deformation can change with rotational frequency, total Routhian surfaces can yield important information on the evolution of collectivity within and between excited bands. Calculations were performed for ${ }^{113}$ I using the model detailed in Ref. [26]. The results showed that the valence neutrons do not contribute significantly to the wave functions of states with excitation energies below $1.6 \mathrm{MeV}$.

Figure 11 shows the calculated surface for an excited state at $690 \mathrm{keV}$ which is minimized at $\beta_{2}=0.18, \gamma=7.4^{\circ}$. The calculated excitation energy and deformation parameter for this configuration are in good agreement with those of the $11 / 2^{+}$

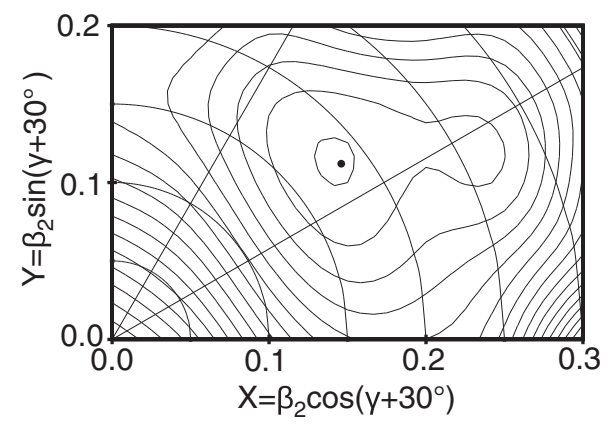

FIG. 11. Results from configuration-constrained total Routhian surface calculations for the $11 / 2^{+}$state in ${ }^{113}$ I. The calculated surface shows a minimum at $\beta_{2}=0.18, \gamma=7.4^{\circ}$ for the $11 / 2^{+}$state at an excitation energy of $690 \mathrm{keV}$ with a configuration of $1 / 2^{+}$[420]. state determined in this work, namely, $E_{x}=753.9(9) \mathrm{keV}$ and $\beta_{2}=0.16(1)$ for $K=1 / 2$ or $\beta_{2}=0.17(2)$ for $K=3 / 2$. The calculated configuration for this state is $1 / 2^{+}[420]$ which, assuming this is the $11 / 2^{+}$state, is consistent with the configurations chosen for the rigid-rotor-model analysis discussed earlier.

Previous TRS calculations by Paul et al. [27] stated that the low-spin $(-,-1 / 2)$ configuration with deformation parameters $\beta=0.19$ and $\gamma \sim+7^{\circ}$ could be associated with a band built on the $\pi h_{11 / 2}, 1 / 2^{-}$[550] orbital and was identified with the negative-parity band built upon on the $11 / 2^{-}$state. The present TRS calculations could not produce a one-quasiparticle, $11 / 2^{-}$bandhead state with an excitation energy of around $1 \mathrm{MeV}$. The $1 / 2^{-}$[550] configuration resided at an excitation energy of $468 \mathrm{keV}$ without cranking which was pushed up to $560 \mathrm{keV}$ once cranked. Three-quasiparticle configurations all had excitation energies in excess of $2 \mathrm{MeV}$, at least double the experimental value of $1017 \mathrm{keV}$ for the $11 / 2^{-}$state.

It is well known that $E 1$ transitions in medium mass nuclei are often hindered [28]; for example, the $1^{+} \rightarrow 2^{-}$ $E 1$ transition in ${ }^{120} \mathrm{I}$ has a reduced transition probability of $5.3(4) \times 10^{-4}$ W.u. It may be expected that both the $388 \mathrm{k}$ and $263 \mathrm{keV}$ decays would be hindered due to the configuration changes taking place; however, a larger hindrance may be expected for a stretched decay from a deformed collective state to a shell-model-like configuration than for an unstretched decay to a prolately deformed state. Figure 11 shows a slight $\gamma$ softness for the $11 / 2^{+}$state which could result in a fairly mixed configuration for this state. This mixing could possibly increase the overlap between the initial and final state wave functions thus reducing any potential decay hindrance. Therefore, it is still not fully clear why similar E1 strengths have been observed for decays from the $11 / 2^{-}$ state to apparently dissimilar, competing structures. Further lifetime measurements in neighboring nuclei, for example ${ }^{111} \mathrm{I}$, are required to help shed light on this issue.

\section{CONCLUSIONS}

Excited state lifetimes have been measured for the first time for the low-lying positive-parity $9 / 2^{+}, \tau=28(4)$ ps and the $11 / 2^{+}, \tau=3.7(7)$ ps states in ${ }^{113} \mathrm{I}$. The lifetime of the yrast negative-parity $11 / 2^{-}$has been remeasured with improved accuracy. Electric quadrupole reduced transition probabilities have been determined for the newly measured states and the large $B\left(E 2: 11 / 2^{+} \rightarrow 7 / 2^{+}\right)=209(39)$ W.u. for the $11 / 2^{+}$state indicates a high degree of collectivity. Using the rigid rotor model, an associated quadrupole deformation of $\beta_{2}=0.17(2)$ or $\beta_{2}=0.16(1)$ was determined if $K=3 / 2$ or $K=1 / 2$, respectively, depending on the choice of Nilsson orbital at the Fermi surface. In contrast, the smaller partial $B\left(E 2: 9 / 2^{+} \rightarrow 5 / 2^{+}\right)=32(5)$ W.u. for the $690 \mathrm{keV}$ decay from the $9 / 2^{+}$state indicates far less collectivity present in the wave function. Shell-model calculations using an effective interaction based on the CD-Bonn nucleon-nucleon potential show good agreement with the experimental low-energy level scheme. The theoretical $B\left(E 2: 9 / 2^{+} \rightarrow 5 / 2^{+}\right)=45$ W.u. 
is in agreement (within $3 \sigma$ ) with the experimentally determined value from this work. The intrinsic quadrupole moment for the $9 / 2^{+}$state was calculated to be $Q_{0}=$ $-0.12 e \mathrm{~b}$, highlighting small quadrupole deformation. Using the remeasured lifetime value for the $11 / 2^{-}$state, electric dipole reduced transition probabilities were determined for the two decays observed to feed the $11 / 2^{+}$and $9 / 2^{+}$states from the $11 / 2^{-}$state. A remarkable similarity between the $B\left(E 1: 11 / 2^{-} \rightarrow 9 / 2^{+}\right)=5.5(5) \times 10^{-4}$ W.u. and the $B\left(E 1: 11 / 2^{-} \rightarrow 11 / 2^{+}\right)=4.9(5) \times 10^{-4}$ W.u. was observed. Configuration-constrained total Routhian surface calculations were performed, the results of which showed that the $11 / 2^{+}$state was slightly $\gamma$ soft. However, this apparent $\gamma$ softness for the $11 / 2^{+}$state does not provide an explanation for the observed similarity between the strengths of the decays depopulating the $11 / 2^{-}$state to apparently dissimilar, competing structures.

\section{ACKNOWLEDGMENTS}

DPUNS was designed and built as a part of a collaboration between the University of Manchester, University of Liverpool, University of Köln and JYFL under STFC Contract No. ST/G008787/1. This work has been supported by the EU 6th Framework Programme, "Integrating Infrastructure Initiative Transnational Access", Contract No. 506065 (EURONS), and by the Academy of Finland under the Finnish Centre of Excellence Programme 2006-2011 (Nuclear and Accelerator Based Physics Programme at JYFL). The authors acknowledge the support of GAMMAPOOL for the loan of the Jurogam-II detectors. T.B., B.C. and M.D. acknowledge the support of the Swedish Research Council under Grants No. 621-2010-3694 and No. 621-2010-4723 and the Nordic Infrastructure support under NordForsk (Project No. 070315). T.G. acknowledges the support of the Academy of Finland (Contract No. 131615).
[1] R. Machleidt, Phys. Rev. C 63, 024001 (2001).

[2] A. Banu, J. Gerl, C. Fahlander, M. Górska, H. Grawe, T. R. Saito, H.-J. Wollersheim, E. Caurier, T. Engeland, A. Gniady et al., Phys. Rev. C 72, 061305 (2005).

[3] J. Cederkäll, A. Ekström, C. Fahlander, A. M. Hurst, M. HjorthJensen, F. Ames, A. Banu, P. A. Butler, T. Davinson, U. D. Pramanik et al., Phys. Rev. Lett. 98, 172501 (2007).

[4] C. Vaman, C. Andreoiu, D. Bazin, A. Becerril, B. A. Brown, C. M. Campbell, A. Chester, J. M. Cook, D. C. Dinca, A. Gade et al., Phys. Rev. Lett. 99, 162501 (2007).

[5] A. Ekström, J. Cederkäll, C. Fahlander, M. Hjorth-Jensen, F. Ames, P. A. Butler, T. Davinson, J. Eberth, F. Fincke, A. Görgen et al., Phys. Rev. Lett. 101, 012502 (2008).

[6] T. Bäck, C. Qi, B. Cederwall, R. Liotta, F. Ghazi Moradi, A. Johnson, R. Wyss, and R. Wadsworth, Phys. Rev. C 87, 031306 (2013).

[7] G. Guastalla, D. D. DiJulio, M. Górska, J. Cederkäll, P. Boutachkov, P. Golubev, S. Pietri, H. Grawe, F. Nowacki, K. Sieja et al., Phys. Rev. Lett. 110, 172501 (2013).

[8] T. Bäck, C. Qi, B. Cederwall, R. Liotta, F. Ghazi Moradi, A. Johnson, R. Wyss, and R. Wadsworth, Phys. Scr. T 150, 014003 (2012).

[9] K. Starosta, C. J. Chiara, D. B. Fossan, T. Koike, D. R. Lafosse, G. J. Lane, J. M. Sears, J. F. Smith, A. J. Boston, P. J. Nolan et al., Phys. Rev. C 64, 014304 (2001).

[10] P. Petkov, A. Dewald, A. Fitzler, T. Klug, G. de Angelis, E. Farnea, A. Gadea, R. Isocrate, N. Marginean, D. R. Napoli et al., Phys. Rev. C 67, 054306 (2003).

[11] M. G. Procter, D. M. Cullen, C. Scholey, P. Ruotsalainen, L. Angus, T. Bäck, B. Cederwall, A. Dewald, C. Fransen, T. Grahn et al., Phys. Lett. B 704, 118 (2011).

[12] T. K. Alexander and J. S. Forster, Advances in Nuclear Physics, Vol. 10 (Plenum, New York, 1978).

[13] M. J. Taylor, D. M. Cullen, A. J. Smith, A. McFarlane, V. Twist, G. A. Alharshan, M. G. Procter, T. Braunroth, A. Dewald, E. Ellinger et al., Nucl. Instrum. Methods Phys. Res. A 707, 143 (2013).
[14] P. T. Greenlees, N. Amzal, J. E. Bastin, E. Bouchez, P. A. Butler, A. Chatillon, O. Dorvaux, S. Eeckhaudt, K. Eskola, B. Gall et al., AIP Conf. Proc. 764, 237 (2005).

[15] M. Leino, J. Aysto, T. Enqvist, P. Heikkinen, A. Jokinen, M. Nurmia, A. Ostrowski, W. H. Trzaska, J. Uusitalo, K. Eskola et al., Nucl. Instrum. Methods Phys. Res. B 99, 653 (1995).

[16] J. Sarén, J. Uusitalo, M. Leino, and J. Sorri, Nucl. Instrum. Methods Phys. Res. A 654, 508 (2011).

[17] I. Lazarus, E. E. Appelbe, P. A. Butler, P. J. Coleman-Smith, J. R. Cresswell, S. J. Freeman, R. D. Herzberg, I. Hibbert, D. T. Joss, S. C. Letts et al., IEEE Trans. Nucl. Sci. 48, 567 (2001).

[18] A. Dewald, S. Harissopulos, and P. von Brentano, Z. Phys. A: Atomic Nuclei 334, 163 (1989).

[19] A. Dewald, O. Möller, and P. Petkov, Prog. Part. Nucl. Phys. 67, 786 (2012).

[20] P. Petkov, A. Dewald, and P. von Brentano, Nucl. Instrum. Methods Phys. Res. A 457, 527 (2001).

[21] B. A. Brown and W. D. M. Rae, MSU-NSCL Rep., 2007 (unpublished).

[22] B. A. Brown, N. J. Stone, J. R. Stone, I. S. Towner, and M. Hjorth-Jensen, Phys. Rev. C 71, 044317 (2005).

[23] R. Machleidt, F. Sammarruca, and Y. Song, Phys. Rev. C 53, R1483 (1996).

[24] A. Bohr and B. R. Mottelson, Nuclear Structure, Vol. 2 (Benjamin, New York, 1975).

[25] T. Kibédi, T. W. Burrows, M. B. Trzhaskovskaya, P. M. Davidson, and C. W. Nestor, Nucl. Instrum. Methods Phys. Res. A 589, 202 (2008).

[26] X. M. Fu, F. R. Xu, J. C. Pei, C. F. Jiao, Y. Shi, Z. H. Zhang, and Y. A. Lei, Phys. Rev. C 87, 044319 (2013).

[27] E. S. Paul, C. W. Beausang, S. A. Forbes, S. J. Gale, A. N. James, P. M. Jones, M. J. Joyce, R. M. Clark, K. Hauschild, I. M. Hibbert et al., Phys. Rev. C 48, R490 (1993).

[28] P. M. Endt, At. Data Nucl. Data Tables 26, 47 (1981). 